\title{
Estudo sobre aplicação de campo magnético no tratamento de águas para consumo humano
}

\section{Study on application of magnetic field in the treatment of water for human consumption}

\author{
Data de entrada: \\ 25/01/2019 \\ - Data de aprovação: \\ 06/03/2019
}

Matheus Pinheiro Massaut ${ }^{1 *}$ | Ramon Lucas Dalsasso ${ }^{1}$

DOI: https://doi.org/10.36659/dae.2020.056

ORCID ID

Massaut MP (D) https://orcid.org/0000-0002-4188-5824

Dalsasso RL (1D) https://orcid.org/0000-0002-5443-6374

\section{Resumo}

No âmbito do tratamento de águas, busca-se sempre o avanço do conhecimento de novos métodos que não requerem extensão de plantas existentes, construção de reatores e que não gerem despesas excessivas. Este artigo apresenta um estudo sobre a aplicação de campo magnético no tratamento de águas para consumo humano, analisando o comportamento dos parâmetros turbidez e cor aparente. 0 sistema de tratamento elaborado nesta pesquisa possui as características de filtração direta, sendo composto pelas etapas de coagulação e filtração. Um gerador de campo magnético foi construído e instalado para magnetizar a água antes e após a aplicação de coagulante. A partir da exposição de $8 \mathrm{mT}$, quando exposto campo magnético associado a aplicação de coagulante químico, foi possível verificar o auxílio do campo magnético na remoção de turbidez e cor aparente.

Palavras-chave: Tratamento Magnético das Águas. Turbidez. Cor Aparente. Campo magnético. Tratamento de Água.

\section{Abstract}

In the field of water treatment, we always seek to advance our knowledge of new methods that do not require the extension of existing plants, construction of reactors and do not generate excessive expenses. This paper presents a study on the application of magnetic field in the treatment of waters for human consumption, analyzing the behavior of the parameters turbidity and apparent color. The treatment system elaborated in this research has the characteristics of direct filtration, being composed by the steps of coagulation and filtration. A magnetic field generator was built and installed to magnetize the water before and after coagulant application. From the exposure of $8 \mathrm{mT}$, when exposed magnetic field associated with application of chemical coagulant, it was possible to verify the aid of the magnetic field in the removal of turbidity and apparent color.

Keywords: Magnetic Water Treatment. Turbidity. Apparent Color. Magnetic field. Water Treatment.

\footnotetext{
1 Universidade Federal de Santa Catarina - Florianópolis - Santa Catarina - Brasil.

* Autor correspondente: mpmassautahotmail.com.
} 


\section{INTRODUÇÃO}

Um dos objetivos do milênio, almejado pela cúpula da Organização Mundial de Saúde (OMS), é a disponibilidade de água para consumo humano em quantidade e qualidade equivalente. Esse desafio é maior em países em ascensão, regiões naturalmente áridas e polos agroindustriais emergentes, onde a demanda por água é fundamental para a produtividade e o desenvolvimento econômico (MOLINA et al, 2016).

A tecnologia de tratamento a ser adotada depende da qualidade da água bruta, dos critérios de qualidade para consumo humano e das características da comunidade a ser beneficiada. Do ponto de vista tecnológico, água de qualquer qualidade pode ser, em princípio, transformada em água potável (DI BERNARDO et al., 1999).

Praticamente toda água que se encontra na natureza, principalmente em corpos de água superficiais, demanda tratamento para ser utilizada no abastecimento público. Isso se deve à diminuição da qualidade das águas, reflexo das diversas atividades antropogênicas da era contemporânea. Além dos poluentes tradicionais, contaminantes emergentes, tais como pesticidas, fármacos, disruptores endócrinos e seus metabólitos, cosméticos e nanopartículas surgem como novos desafios para os sistemas convencionais de produção de água potável (HESPANHOL, 2015; BENOTTI et al, 2009). Com isso, a queda da qualidade da água bruta impacta diretamente em maior consumo de produtos químicos e geração de resíduos ao final do processo de tratamento (OLIVEIRA, 2010).

Possíveis impactos ambientais provenientes do uso de coagulantes à base de sais inorgânicos são constantemente discutidos. Os resíduos primeiramente são formados nos decantadores das estações de tratamento de água como resultado dos processos de coagulação e floculação e junto com a água de lavagem dos filtros (TEIXEIRA, MELO e SILVA, 2005; GUIMARÃES e PÁDUA, 2005). Esses resíduos, ricos em hidróxidos metálicos não biodegradáveis, apresen- tam grande potencial de poluição e contaminação ao meio ambiente (SCALIZE e DI BERNARDO, 2010). Outra preocupação é o residual de alumínio na água tratada para consumo humano. Walton (2013) aponta para os malefícios da exposição humana a esse metal, correlacionando-o com doenças neurodegenerativas, como, por exemplo, o Alzheimer.

Frente à problemática exposta, buscam-se meios que aumentem a segurança e a eficiência do tratamento e a qualidade das águas relacionando o avanço tecnológico e econômico. Com esse objetivo, uma alternativa que tem sido avaliada é a aplicação de campos magnéticos para assistir os processos do tratamento de água de forma potencialmente eficaz (ZAIDI, et al. 2014). O magnetismo pode afetar as propriedades físicas dos contaminantes na água de forma a potencializar sua manipulação e eliminação mais facilmente.

Segundo Zaidi et al. (2014), a aplicação de campo magnético no tratamento das águas tem a capacidade de melhorar o desempenho físico em termos de separação sólido-líquido, principalmente por meio da coagulação das partículas coloidais. Uma das vantagens da implementação de campos magnéticos em sistemas de tratamento de água é que se trata de uma tecnologia verde. Segundo Zularisame e Johan (2007), no caso do uso de ímãs permanentes, não há necessidade de reposição e compra de materiais e gastos com eletricidade. 0 emprego e a instalação de dispositivos para geração de campo magnético no sistema de tratamento de água são considerados simples e econômicos (ZAIDI et al. 2014).

Por tais razões, este trabalho teve como objetivo principal estudar a aplicação do campo magnético no tratamento de águas para abastecimento público, analisando a capacidade do campo magnético de auxiliar a remoção de turbidez e cor aparente. 0 estudo foi realizado em um sistema em escala piloto, composto por um gerador de campo magnético, unidade de coagulação e filtração. 


\section{METODOLOGIA}

As investigações experimentais ocorreram no Laboratório de Águas da Lagoa do Peri (LALP), localizado junto à Estação de Tratamento de Águas da Companhia Catarinense de Águas e Saneamento (CASAN), administrado e mantido pela
Universidade Federal de Santa Catarina (UFSC), no período entre junho e outubro de 2018.

Para a realização dos experimentos foi construído um sistema piloto, conforme esquematizado na Fig. 1.

Figura 1 - Esquema sistema piloto.

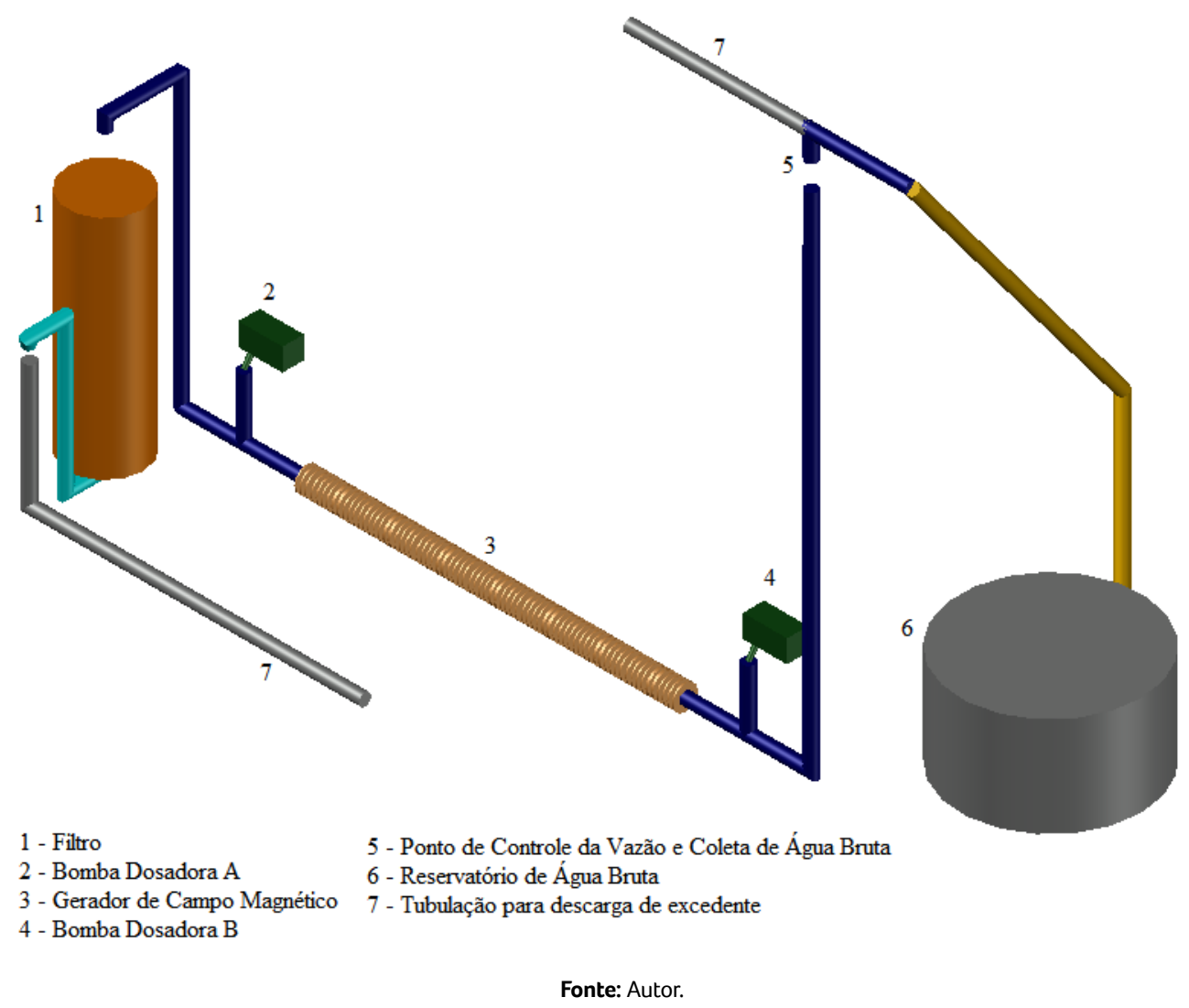

Filtro: foi confeccionado em tubo cilíndrico de PVC de $200 \mathrm{~mm}$ de diâmetro, 1,20 m de altura, com volume total de $37,7 \mathrm{~L}$. A espessura da camada filtrante foi fixada em $60 \mathrm{~cm}$, e o diâmetro dos grãos, entre $0,42-0,85 \mathrm{~mm}$, sendo o valor do diâmetro efetivo igual a $0,53 \mathrm{~mm}$. O filtro, de escoamento descendente, foi operado com vazão de 70 L.h ${ }^{-1}$, sendo a taxa de filtração igual a $36 \mathrm{~m} \cdot \mathrm{d}^{-1}$. Segundo Mondardo (2009), os valores de turbidez, cor aparente e $\mathrm{pH}$ das águas da Lagoa do Peri encontramse entre, respectivamente: 1,20 e 7,61 NTU - 15 e $95 \mathrm{uH}-6,44$ e 7,75. $\mathrm{O}$ coagulante utilizado na pesquisa foi o hidróxicloreto de alumínio (PAC), 10,5\% de $\mathrm{Al}_{2} \mathrm{O}_{3}$. Para aplicar o coagulante, utilizou-se bomba dosadora da marca Exatta, modelo 1201.

Gerador de campo magnético: o campo magnético para os ensaios foi gerado por um sistema de bobinas com características indicadas na Tabela 1. Utilizou-se como suporte uma tubulação de aço galvanizado com diâmetro externo igual a 48,5 mm e interno, 41,5 mm. Em torno da tubulação, passou-se fio de cobre do tipo esmaltado, 14 AWG, e também foi utilizada uma fonte elétrica de $12 \mathrm{~V}$ e $12 \mathrm{~A}$. A Tabela 1 apresenta as características gerais do dispositivo. 
Tabela 1 - Características gerais da bobina

\begin{tabular}{|c|c|c|c|}
\hline Características & \multicolumn{3}{|c|}{ Bobina } \\
\hline & $\mathbf{1}$ & $\mathbf{2}$ & $\mathbf{3}$ \\
\hline Comprimento $(\mathbf{m})$ & 0,50 & 1,00 & 1,50 \\
\hline Número de espiras & 1176 & 2353 & 3529 \\
\hline Resistência $(\mathbf{\Omega})$ & 1,50 & 3,00 & 4,50 \\
\hline Corrente elétrica $(\mathbf{A})$ & 8 & 4 & 3 \\
\hline Campo magnético $(\mathbf{m T})$ & 24 & 12 & 8 \\
\hline Tempo de Contato $(\mathbf{s})$ & 72 & 143 & 215 \\
\hline
\end{tabular}

Os ensaios foram organizados combinando-se todas as variáveis possíveis (intervalo de concentração de coagulante e intensidade de campo magnético) e, também, levando-se em conta três configurações distintas no sistema piloto: 0 , A e B.

A diferença entre as configurações $0, A$ e B é, basicamente, o que ocorre antes de a água ser encaminhada para o filtro:

- Configuração 0: tratamento da água sem coagulante, tendo apenas o efeito da magnetização como auxílio;

- Configuração A: tratamento da água com coagulante; neste caso, primeiramente, a água recebe a magnetização e, posteriormente, a aplicação de coagulante;

- Configuração B: tratamento da água com coagulante; neste caso, primeiramente, a água recebe a aplicação de coagulante e, posteriormente, a magnetização.

A concentração ótima de coagulante utilizado nos experimentos foi determinada em ensaios de jar test. O equipamento utilizado é da marca Nova Ética, modelo LDB 310. No equipamento foram acoplados filtros de laboratório de areia (FLAs), sendo a altura da camada de areia igual a $15 \mathrm{~cm}$, diâmetro dos grãos entre 0,42-0,85 $\mathrm{mm}$ e diâmetro efetivo de 0,53 mm, e a carga hidráulica de filtração, constante, igual a $11 \mathrm{~cm} .0$ gradiente de velocidade de mistura rápida adotado foi de $1200 \mathrm{~s}-1$ ( $400 \mathrm{rpm}$ ), sendo o tempo de mistura rápida igual a $30 \mathrm{~s}$ e o gradiente de velocidade após a mistura rápida igual a $150 \mathrm{~s}^{-1}$ ( 45 rpm). Por fim, a taxa de filtração foi de aproximadamente $100 \mathrm{~m}^{3} \cdot\left(\mathrm{m}^{2} \cdot \mathrm{d}\right)^{-1}$. Esses parâmetros foram adotados a partir dos estudos realizados por Dalsasso (2005) e Melo Filho (2006), levando-se em consideração a turbidez da água bruta e a dosagem de coagulante.

Após a determinação da concentração ótima de coagulante, realizaram-se os ensaios no sistema piloto. Em todas as situações foram testadas as seguintes intensidades de campo magnético: $0,8,12$ e $24 \mathrm{mT}$. Além da concentração ótima de coagulante, também foram utilizadas outras concentrações de coagulante. Fixou-se o valor da concentração ótima de coagulante encontrado, nomeando-o de "100 \%”, e escolheu-se utilizar também nos ensaios experimentais um intervalo de valores a partir da concentração ótima. Em todas as situações foram testadas as seguintes concentrações de coagulante: $100 \%, 80 \%, 60 \%, 40 \%$, $20 \%$ e $0 \%$. Essa escolha se deu com o objetivo de analisar a capacidade da magnetização de compensar a redução na concentração de coagulante.

Todos os ensaios foram repetidos três vezes, constituindo, assim, dados em triplicata. Foram observados os valores de Turbidez e Cor Aparente, da água bruta e tratada. A coleta de água bruta ocorreu no ponto de controle do sistema, no ponto 5 da Fig. 1; já a água tratada foi coletada na saída do filtro. Foram coletadas amostras de água filtrada após 30 minutos do início dos ensaios para a análise dos parâmetros Turbidez e Cor Aparente. 


\subsection{Análise Estatística}

Foram elaborados gráficos de distribuição para análise dos resultados. Fez-se o teste T de Student para investigar a relação entre os ensaios com e sem campo magnético para os resultados obtidos em turbidez. Antes foi verificada a normalidade dos dados utilizando o coeficiente do teste de Shapiro-Wilk, sendo que, para os dados serem considerados normais, o coeficiente deve ser maior do que 0,05 . Utilizaram-se os seguintes conjuntos de hipóteses para o teste T de Student:

i: Conjunto 1:

HO: Não há relação entre as variáveis, logo se aceita que as médias são semelhantes;

H1: Há relação entre as variáveis, portanto suas médias são diferentes.

i: Conjunto 2:

HO: Não há relação entre as variáveis, então se aceita que as médias são semelhantes;

H1: Há relação entre as variáveis, sendo que média da primeira categoria é maior que a média da segunda. i: Conjunto 3:

HO: Não há relação entre as variáveis, logo se aceita que as médias são semelhantes;

H1: Há relação entre as variáveis, sendo a média da categoria 1 menor que a média da categoria 2 .

- HO: $\mathrm{p}$-value $\geq \alpha / 2$, caso contrário $\mathrm{H} 1$ (Conjunto 1); ou,

- H1: $\mathrm{p}$-value $\leq \alpha$, caso contrário H1 (Categoria 2 e Categoria 3).

Para se decidir estatisticamente entre as duas hipóteses ( $\mathrm{HO}$ e $\mathrm{H} 1$ ), são comparados a probabilidade de significância p-value e o nível de significância ( $\alpha$ ) adotado. Para o trabalho em questão, foi adotado o valor de $5 \%$.

\section{RESULTADOS E DISCUSSÃO}

Determinação da concentração ótima de coagulante - ensaios de jar test

Os resultados obtidos nos ensaios de jartest encontram-se na Fig. 2.

Figura 2 - Diagrama de Coagulação

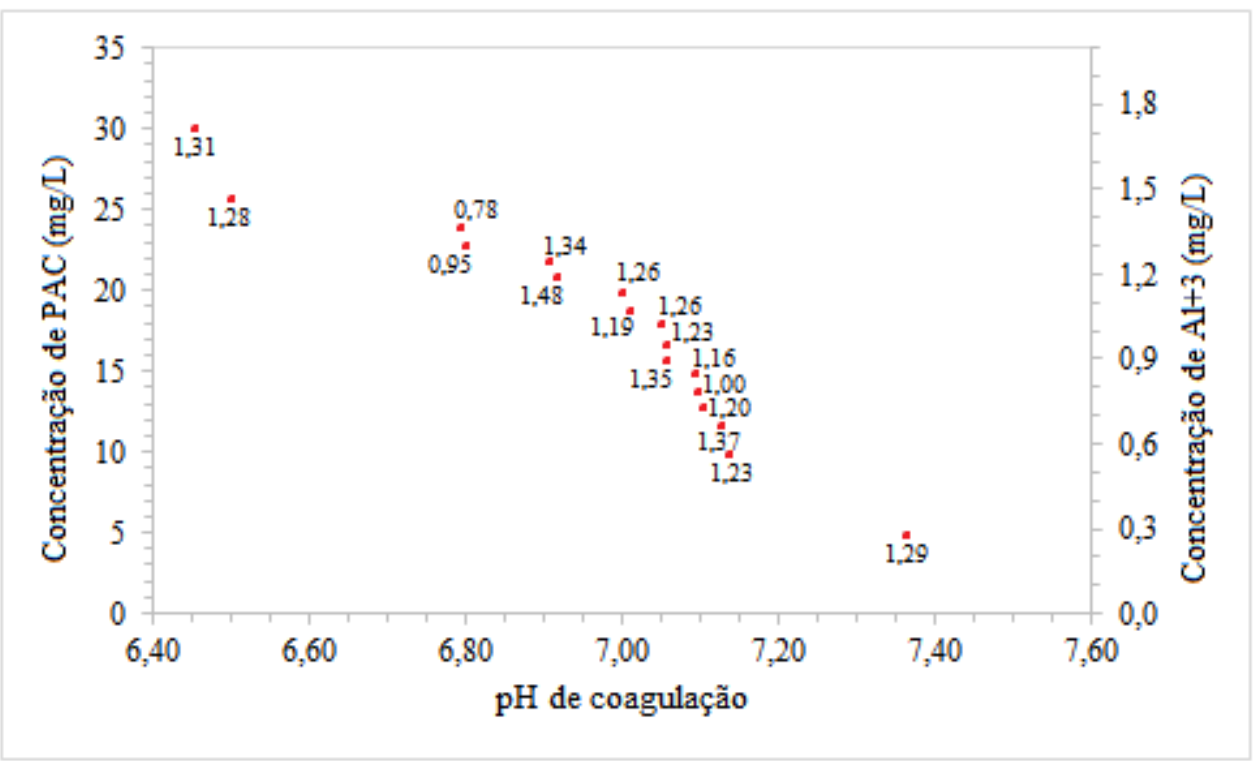


A partir dos resultados obtidos, é possível concluir que os ensaios que apresentaram os valores mais baixos de turbidez foram aqueles cujas concentrações de coagulante e $\mathrm{pH}$ de coagulação estavam entre, respectivamente, 23 e $25 \mathrm{mg}^{\mathrm{L}}{ }^{-1}$ 6,80 e 6,79. Di Bernardo et al. (2003) em pesquisa com as águas da Lagoa do Peri, escolheram trabalhar com a concentração de 22,0 mg.L ${ }^{-1}$. Tendo em vista os resultados obtidos experimentalmente e as contribuições dos pesquisadores, escolheu-se adotar a concentração de $24 \mathrm{mg} \cdot \mathrm{L}^{-1}$ como a ideal para remoção de turbidez. Assim, fixouse como $100 \%$ a concentração de $24,0 \mathrm{mg} \cdot \mathrm{L}^{-1}$. Dessa forma, os valores de $80 \%, 60 \%, 40 \%$ e $20 \%$ utilizados nos ensaios foram, respectivamente $19,2-14,4-9,6$ e $4,8 \mathrm{mg} \cdot \mathrm{L}^{-1}$.

\subsection{Ensaios no sistema piloto}

As tabelas com os resultados encontram-se no Apêndice.

\subsubsection{Configuração 0}

A Fig. 3 apresenta, respectivamente, a distribuição gráfica dos resultados obtidos nos ensaios da Configuração 0 para os parâmetros de Turbidez e Cor Aparente.

Figura 3 - Turbidez e Cor Aparente da Configuração 0
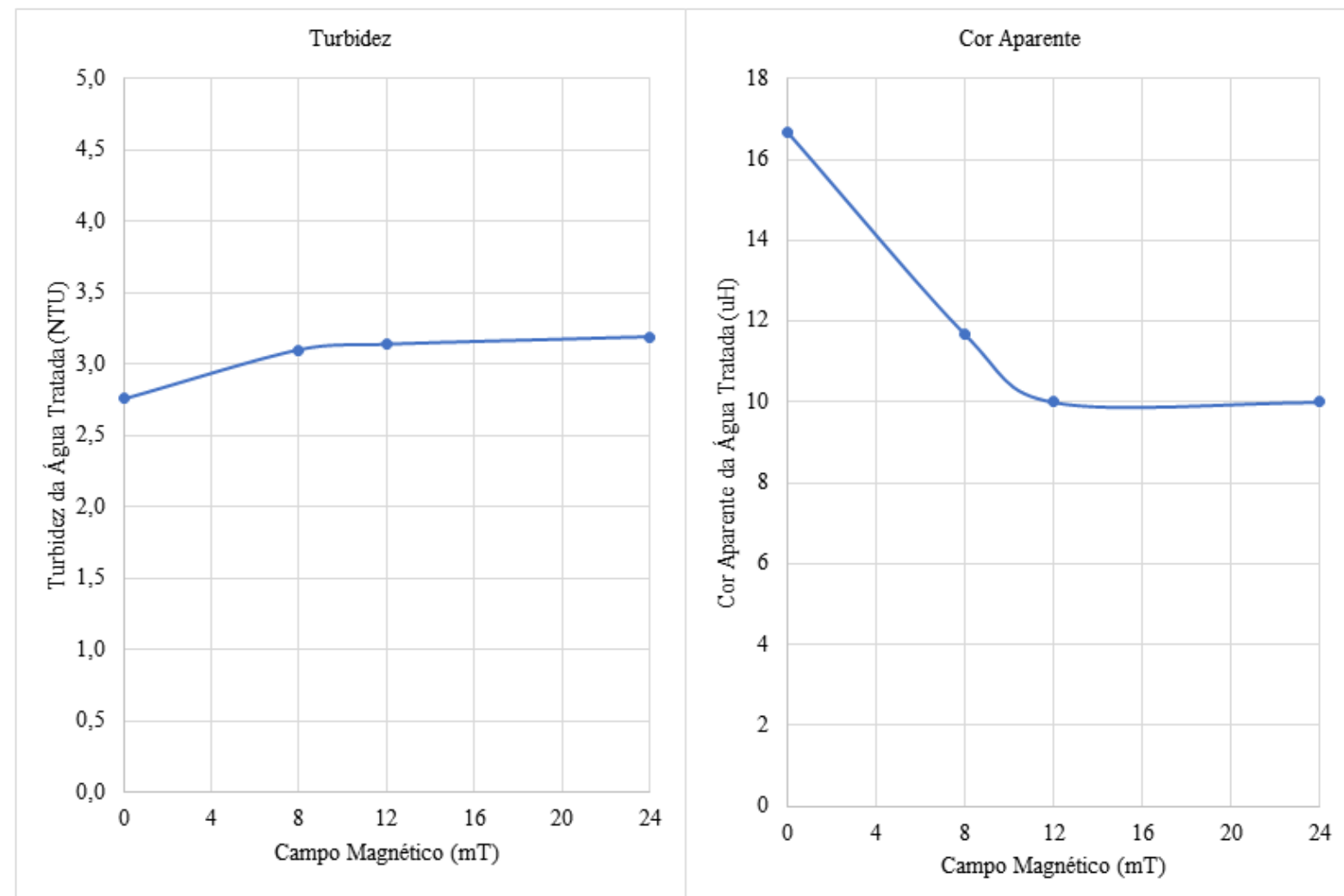

Os resultados obtidos nos ensaios com a Configuração 0 não demonstraram com clareza que a ação do campo magnético poderia auxiliar no tratamento das águas. A partir dos resultados de turbidez é possível observar que as médias da turbidez da água tratada nos ensaios com campo magnético de 8,12 e $24 \mathrm{mT}$, respectivamente, $3,10-3,14$ e 3,19 NTU, foram superiores à 
média da turbidez da água tratada sem campo magnético, 2,76 NTU. Com relação à Cor Aparente, nota-se que houve redução dos valores a partir da exposição de campo magnético de 8 $\mathrm{mT}$, apresentando em 12 e $24 \mathrm{mT}$ os valores mais baixos, $10 \mathrm{uH}$. Assim, com apenas o tratamento com campo magnético substituindo por completo o papel do coagulante químico, não é possível afirmar que seria suficiente para o tratamento de águas para consumo humano.

\subsubsection{Configuração $A$}

A Fig. 4 apresenta, respectivamente, a distribuição gráfica dos resultados obtidos nos ensaios da Configuração A para os parâmetros de Turbidez e Cor Aparente.

Figura 4 - Turbidez e Cor Aparente da Configuração A

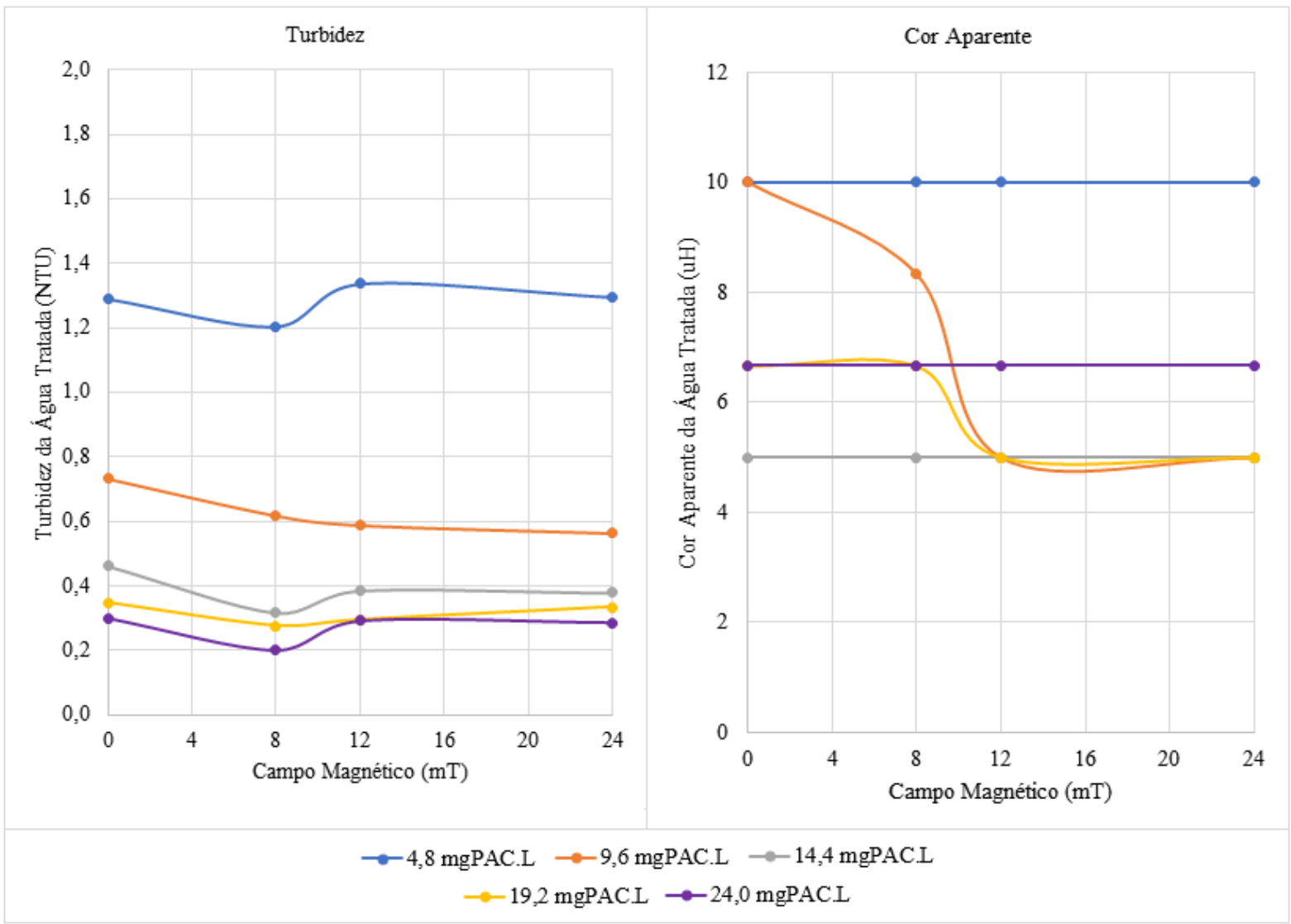

Com relação aos dados obtidos nos ensaios com a Configuração $A$, os resultados apontam para o potencial do campo magnético de melhorar o tratamento de águas. Observa-se que todos os ensaios quando foram expostos ao campo magnético de $8 \mathrm{mT}$ apresentaram uma melhoria em seus resultados, atingindo valores de turbidez in- feriores aos ensaios sem campo magnético. Também é possível observar que quando foi exposto o campo magnético de $24 \mathrm{mT}$, os ensaios em que foram dosados 9,6 - 14,4 - 19,2 e 24,0 mgPAC. $\mathrm{L}^{-1}$ apresentaram turbidez inferior aos ensaios sem campo magnético. A respeito dos resultados obtidos em Cor Aparente, é possível observar que 
apenas nos ensaios em que foram aplicados 9,6 e 19,2 mgPAC.L-1 houve redução de cor aparente na medida em que se aumentou a intensidade de campo magnético. Os valores mais baixos, $5 \mathrm{uH}$, foram verificados nas intensidades de 12 e 24 mT.

\subsubsection{Configuração B}

A Fig. 5 apresenta, respectivamente, a distribuição gráfica dos resultados obtidos nos ensaios da Configuração B para os parâmetros de Turbidez e Cor Aparente.

Figura 5 - Turbidez e Cor Aparente da Configuração B

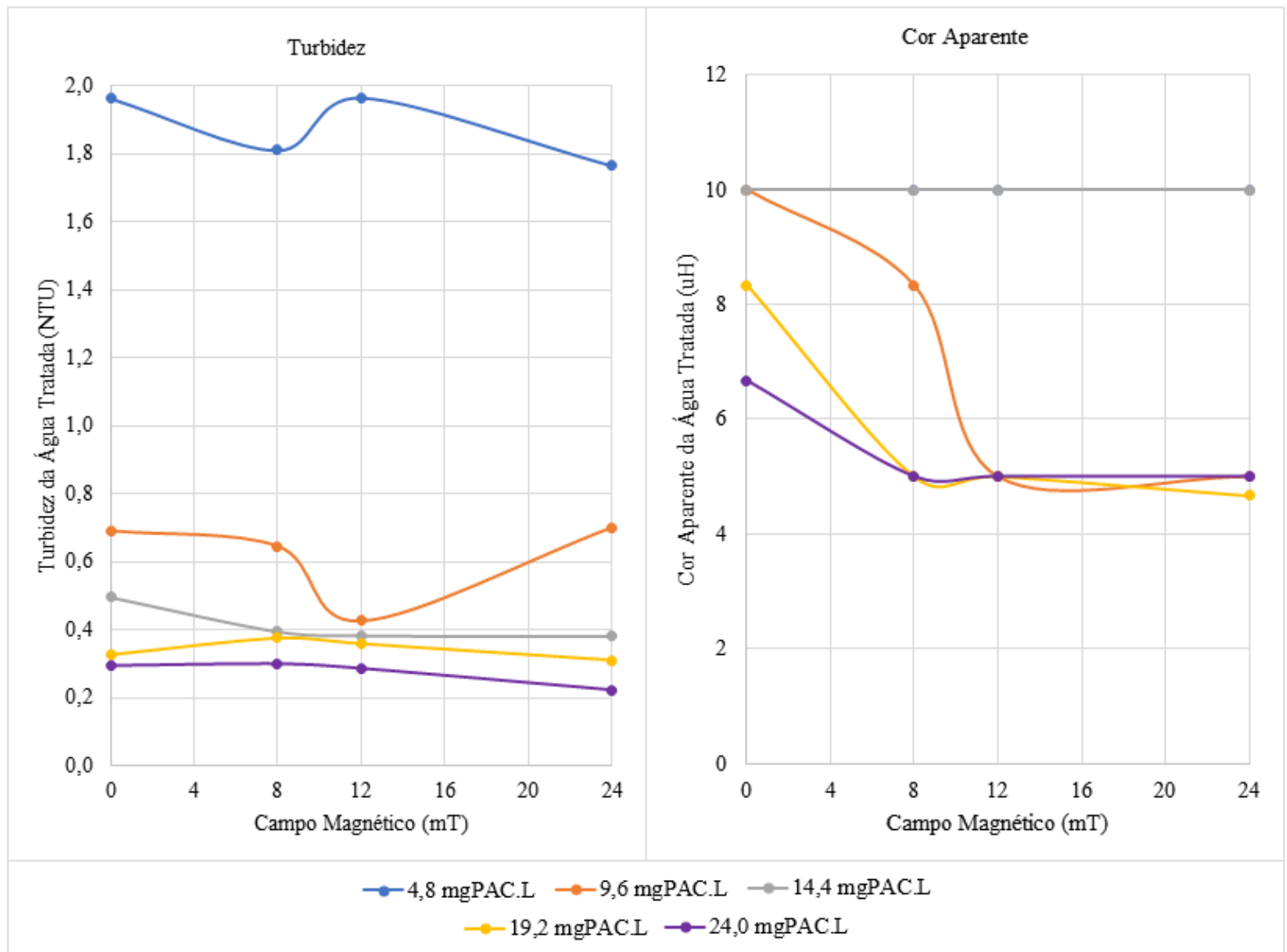

Os resultados alcançados nos ensaios com a Configuração $B$, novamente, evidenciaram a capacidade do campo magnético de auxiliar a remoção de turbidez. Observa-se que os ensaios em que foram aplicados 4,8 - 9,6 - 14,4 e 24,0 mgPAC.L-1, quando exposto campo magnético de $8 \mathrm{mT}$, apresentaram uma melhora, atingindo valores de turbidez da água tratada inferiores aos ensaios sem campo magnético. Da mesma forma, os ensaios em que foram aplicados 4,8 - 14,4 19,2 e 24,0 mgPAC.L-1 , quando exposto cam- po magnético de $24 \mathrm{mT}$, também apresentaram valores de turbidez da água tratada inferiores aos ensaios sem campo magnético. A respeito dos resultados obtidos para Cor Aparente, verifica-se que nos ensaios em que foram aplicados 9,6 - 19,2 e 24,0 mgPAC. $\mathrm{L}^{-1}$ houve redução de cor aparente na medida em que aumentou a intensidade de campo magnético. Nesses casos, os valores mais baixos também foram observados quando se expuseram os campos magnéticos de 12 e $24 \mathrm{mT}$. 


\subsection{Teste de hipóteses}

Encontram-se a seguir, nas Tabelas 2, 3 e 4, os dados obtidos no teste T de Student, respectivamente das Configurações 0 , A e B.

Tabela 2 - Configuração 0

\begin{tabular}{|c|c|}
\hline \multicolumn{2}{|c|}{ Configuração 0} \\
\hline T de Student & p \\
\hline $0 \mathrm{mT} \times 8 \mathrm{mT}$ & 0,0000 \\
\hline $0 \mathrm{mT} \times 12 \mathrm{mT}$ & 0,0000 \\
\hline $0 \mathrm{mT} \times 24 \mathrm{mT}$ & 0,0000 \\
\hline
\end{tabular}

Tabela 3 - Configuração A

\begin{tabular}{|c|c|}
\hline \multicolumn{2}{|c|}{ Configuração A } \\
\hline [PAC] & $4,8 \mathrm{mg} \cdot \mathrm{L}^{-1}$ \\
\hline T de Student & $\mathbf{p}$ \\
\hline $0 \mathrm{mT} \times 8 \mathrm{mT}$ & 0,5006 \\
\hline $0 \mathrm{mT} \times 12 \mathrm{mT}$ & 0,7002 \\
\hline $0 \mathrm{mT} \times 24 \mathrm{mT}$ & 0,9375 \\
\hline [PAC] & $9,6 \mathrm{mg} \cdot \mathrm{L}^{-1}$ \\
\hline T de Student & p \\
\hline $0 \mathrm{mT} \times 8 \mathrm{mT}$ & 0,1214 \\
\hline $0 \mathrm{mT} \times 12 \mathrm{mT}$ & 0,0930 \\
\hline $0 \mathrm{mT} \times 24 \mathrm{mT}$ & 0,0018 \\
\hline [PAC] & $14,4 \mathrm{mg} \cdot \mathrm{L}^{-1}$ \\
\hline T de Student & p \\
\hline $0 \mathrm{mT} \times 8 \mathrm{mT}$ & 0,0407 \\
\hline $0 \mathrm{mT} \times 12 \mathrm{mT}$ & 0,0181 \\
\hline $0 \mathrm{mT} \times 24 \mathrm{mT}$ & 0,0451 \\
\hline [PAC] & $19,2 \mathrm{mg} \cdot \mathrm{L}^{-1}$ \\
\hline T de Student & p \\
\hline $0 \mathrm{mT} \times 8 \mathrm{mT}$ & 0,0502 \\
\hline $0 \mathrm{mT} \times 12 \mathrm{mT}$ & 0,1350 \\
\hline $0 \mathrm{mT} \times 24 \mathrm{mT}$ & 0,2844 \\
\hline [PAC] & $24,0 \mathrm{mg} \cdot \mathrm{L}^{-1}$ \\
\hline T de Student & $\mathbf{p}$ \\
\hline $0 \mathrm{mT} \times 8 \mathrm{mT}$ & 0,1265 \\
\hline $0 \mathrm{mT} \times 12 \mathrm{mT}$ & 0,7750 \\
\hline $0 \mathrm{mT} \times 24 \mathrm{mT}$ & 0,6897 \\
\hline
\end{tabular}

Realizaram-se ao todo 33 testes t-Student para verificar a existência de diferenças significativas entre as médias de turbidez da água tratada com e sem campo magnético. Destes, 21 testes resultaram em $\mathrm{p}$-value acima do nível de significância e, com isto, considera-se que não há diferenças significativas entre as médias. Porém
Tabela 4 - Configuração B

\begin{tabular}{|c|c|}
\hline \multicolumn{2}{|c|}{ Configuração B } \\
\hline [PAC] & $4,8 \mathrm{mg} \cdot \mathrm{L}^{-1}$ \\
\hline T de Student & $\mathbf{p}$ \\
\hline $0 \mathrm{mT} \times 8 \mathrm{mT}$ & 0,0253 \\
\hline $0 \mathrm{mT} \times 12 \mathrm{mT}$ & 0,9811 \\
\hline $0 \mathrm{mT} \times 24 \mathrm{mT}$ & 0,0299 \\
\hline [PAC] & $9,6 \mathrm{mg} \cdot \mathrm{L}^{-1}$ \\
\hline T de Student & p \\
\hline $0 \mathrm{mT} \times 8 \mathrm{mT}$ & 0,4194 \\
\hline $0 \mathrm{mT} \times 12 \mathrm{mT}$ & 0,0039 \\
\hline $0 \mathrm{mT} \times 24 \mathrm{mT}$ & 0,7931 \\
\hline [PAC] & $14,4 \mathrm{mg} \cdot \mathrm{L}^{-1}$ \\
\hline T de Student & $\mathbf{p}$ \\
\hline $0 \mathrm{mT} \times 8 \mathrm{mT}$ & 0,0559 \\
\hline $0 \mathrm{mT} \times 12 \mathrm{mT}$ & 0,0061 \\
\hline $0 \mathrm{mT} \times 24 \mathrm{mT}$ & 0,0452 \\
\hline [PAC] & $19,2 \mathrm{mg} \cdot \mathrm{L}^{-1}$ \\
\hline T de Student & p \\
\hline $0 \mathrm{mT} \times 8 \mathrm{mT}$ & 0,0573 \\
\hline $0 \mathrm{mT} \times 12 \mathrm{mT}$ & 0,1354 \\
\hline $0 \mathrm{mT} \times 24 \mathrm{mT}$ & 0,6793 \\
\hline [PAC] & $24,0 \mathrm{mg} \cdot \mathrm{L}^{-1}$ \\
\hline T de Student & $\mathbf{p}$ \\
\hline $0 \mathrm{mT} \times 8 \mathrm{mT}$ & 0,7524 \\
\hline $0 \mathrm{mT} \times 12 \mathrm{mT}$ & 0,6590 \\
\hline $0 \mathrm{mT} \times 24 \mathrm{mT}$ & 0,0854 \\
\hline
\end{tabular}

12 testes resultaram em $p$-value abaixo de 0,05; são eles:

- Configuração 0:

$-0 \mathrm{mT} \times 8 \mathrm{mT}$;

$-0 \mathrm{mT} \times 12 \mathrm{mT}$;

$-0 \mathrm{mT} \times 24 \mathrm{mT}$; 
- Configuração A:

$$
\begin{aligned}
& \text { » 9,6 mgPAC.L-1: } \\
& \text { - } 0 \mathrm{mT} \times 24 \mathrm{mT} \text {; } \\
& \text { »14,4 mgPAC. L-1 } \\
& \text { - } 0 \mathrm{mT} \times 8 \mathrm{mT} \text {; } \\
& \text { - } 0 \mathrm{mT} \times 12 \mathrm{mT} \text {; } \\
& \text { - } 0 \mathrm{mT} \times 24 \mathrm{mT} \text {; }
\end{aligned}
$$

- Configuração B:

$$
\begin{aligned}
& \text { » } 4,8 \mathrm{mgPAC} . \mathrm{L}^{-1} \text { : } \\
& \text { - } 0 \mathrm{mT} \times 8 \mathrm{mT} ; \\
& \text { - } 0 \mathrm{mT} \times 24 \mathrm{mT} ; \\
& \text { » } 9,6 \mathrm{mgPAC} . \mathrm{L}^{-1}: \\
& \text { - } 0 \mathrm{mT} \times 12 \mathrm{mT} ; \\
& \text { » } 14,4 \mathrm{mgPAC} . \mathrm{L}^{-1}: \\
& \text { - } 0 \mathrm{mT} \times 12 \mathrm{mT} ; \\
& \text { - } 0 \mathrm{mT} \times 24 \mathrm{mT} ;
\end{aligned}
$$

Com relação aos resultados dos testes da Configuração 0 , nota-se que o $p$-value das análises é menor do que o nível de significância adotado. Assim, confirma-se que há diferença significativa entre as médias. Considerando que nestes ensaios a turbidez da água filtrada foi menor quando não houve exposição de campo magnético, acredita-se que apenas a exposição de campo magnético não foi suficiente para substituir completamente o coagulante químico. Os demais resultados dos testes de hipóteses que apontaram para p-value abaixo do nível de significância dizem respeito às Configuração $A e$ $B$ onde houve diferença significativa entre as médias, sendo que nestes ensaios a turbidez da água filtrada foi menor quando houve exposição de campo magnético, e, assim, acredita-se que a exposição do campo auxiliou no tratamento das águas potencializando a ação do coagulante.

\section{CONCLUSÃO}

A partir dos resultados obtidos, acredita-se que a utilização de campo magnético no tratamento das águas para consumo humano pode auxiliar na remoção de turbidez e cor aparente. Entretanto, os ensaios realizados não sugerem que aplicação de campo magnético possui a capacidade de substituir por completo os coagulantes químicos, visto que na Configuração 0 os resultados obtidos sem a exposição de campo magnético foram melhores. Contudo, quando exposto campo magnético em associação com a aplicação de coagulante químico, ficou evidenciada a capacidade do campo magnético de conseguir auxiliar o tratamento das águas, visto que houve redução de turbidez e cor aparente na medida em que se aumentou a intensidade de campo magnético.

\section{CONTRIBUIÇÃO DOS AUTORES}

Todos os autores contribuíram de forma igualitária.

\section{REFERÊNCIAS}

BENOTTI, M. J.; TRENHOLM, R. A.; VANDERFORD, B. J.; HOLADY, J. C.; STANFORD, B. D.; SNYDER, S. A. Pharmaceuticals and endocrine disrupting compounds in u.s. Drinking Water. Environmental Science \& Technology 2009, 43, 597. https://doi.org/10.1021/ es801845a

DALSASSO, R. L. Estudo de diferentes materiais para floculação em meio granular, precedendo a filtração rápida descendente no tratamento de água para abastecimento humano. Tese (Doutorado em Engenharia Ambiental) - Universidade Federal De Santa Catarina, 2005. 272 p.: il.

DI BERNARDO, L. (COORD.); MENDES, C. G. N.; BRANDÃO, C. C. S.; SENS, M. L. \& PÁDUA, V. L. Tratamento de água para abastecimento por filtração direta. Rio de Janeiro, ABES, 2003, 498p.

DI BERNARDO, L., BRANDÃO, C. C. S., \& HELLER, L. PROSAB - Programa de Pesquisa em Saneamento Básico. Tratamento de águas de abastecimento por filtração em múltiplas etapas, 1999, 114 p.

DI BERNARDO, L.; SCALIZE, P. S. Estudo da disposição de resíduos gerados em estação de tratamento de água em decantador primário de estação de tratamento de esgoto. In: X SIBESA - Simpósio Ítalo-Brasileiro de Engenharia Sanitária e Ambiental, Maceió, 2010. Anais..

GUIMARÃES, N. C.; PADUA, V. L. Avaliação de parâmetros físico-químicos para recuperação de sulfato de alumínio de resíduos gerados em uma estação de tratamento de água. $23^{\circ}$ Congresso Brasileiro de Engenharia Sanitária e Ambiental, Revista USP 2015, $106 \mathrm{p}$. 
MELO FILHO, L. C. Avaliação da ozonização como pré ou pós-tratamento à filtração direta descendente na remoção de cianobactérias e saxitoxinas. Tese (Doutorado em Engenharia Ambiental) - Universidade Federal de Santa Catarina, 2006. 268 p.

MOLINA, N. F.; RODRÍGUEZ, E. J. M.; ARIZA, C. P. Coagulantes naturales en sistemas de flujo continuo, como sustituto del $\mathrm{Al}_{2}\left(\mathrm{SO}_{4}\right)_{3}$ para clarificación de aguas. producción + limpia 2016 $11,41$.

MONDARDO, R. I. Avaliação da filtração em margem como pré-tratamento à filtração direta descendente na remoção de células de cianobactérias e saxitoxinas. Tese (Doutorado em Engenharia Ambiental) - Universidade Federal de Santa Catarina, 2009, 308 p.

OLIVEIRA, T. F. de.; Tratamento de água para abastecimento público por sistema de separação por membrana de ultrafiltra- ção: estudo de caso na ETA Alto da Boa Vista. Dissertação (Mestrado), Escola Politécnica da Universidade de São Paulo, 2010.

TEIXEIRA, S. T.; MELO, W. J.; SILVA, E. T. Aplicação de lodo da estação de tratamento de água em solo degradado. Brasília, 2005.

WALTON, J. R. Aluminum's involvement in the progression of alzheimer's disease. Journal of Alzheimer's Disease 2013, 35 p, vol 7. https://doi.org/10.3233/jad-121909

ZAIDI, N. S., SOHAILI, J., MUDA, K., \& SILLANPÄÄ, M. Magnetic field application and its potential in water and waste water treatment systems. Separation e purification reviews, 43(3), 206-240, 2014. https://doi.org/10.1080/15422119.2013.794148

ZULARISAM, A.W., FADIL. O., JOHAN, S. Electromagnetic technology on sewage treatment. Tese (Doutorado em Filosofia) - Universiti Teknologi Malaysia, 2007.

\section{APÊNDICE}

\begin{tabular}{|c|c|c|c|c|c|c|c|}
\hline \multicolumn{8}{|c|}{ Configuração 0} \\
\hline \multirow{2}{*}{ Campo } & \multirow{2}{*}{ Triplicata } & \multicolumn{2}{|c|}{ Turbidez (NTU) } & \multicolumn{2}{|c|}{ Cor Aparente (uH) } & \multicolumn{2}{|c|}{ pH } \\
\hline & & Inicial & Final & Inicial & Final & Inicial & Final \\
\hline \multirow{4}{*}{0} & 1 & 6,56 & 2,61 & 30 & 20 & 7,20 & 7,19 \\
\hline & 2 & 8,08 & 2,95 & 40 & 15 & 7,20 & 7,15 \\
\hline & 3 & 6,13 & 2,72 & 25 & 15 & 7,20 & 7,17 \\
\hline & Média & 6,92 & 2,76 & 32 & 17 & 7,20 & 7,17 \\
\hline \multirow{4}{*}{8} & 1 & 9,75 & 3,20 & 35 & 15 & 7,20 & 7,20 \\
\hline & 2 & 8,46 & 3,00 & 30 & 10 & 7,20 & 7,20 \\
\hline & 3 & 9,23 & 3,11 & 25 & 10 & 7,30 & 7,30 \\
\hline & Média & 9,15 & 3,10 & 30 & 12 & 7,23 & 7,23 \\
\hline \multirow{4}{*}{12} & 1 & 8,57 & 3,09 & 25 & 10 & 7,14 & 7,14 \\
\hline & 2 & 8,02 & 3,16 & 30 & 10 & 7,27 & 7,25 \\
\hline & 3 & 7,86 & 3,17 & 25 & 10 & 7,28 & 7,27 \\
\hline & Média & 8,15 & 3,14 & 27 & 10 & 7,23 & 7,22 \\
\hline \multirow{4}{*}{24} & 1 & 9,45 & 2,76 & 25 & 10 & 7,14 & 7,13 \\
\hline & 2 & 8,25 & 3,43 & 25 & 10 & 7,16 & 7,14 \\
\hline & 3 & 6,55 & 3,39 & 25 & 10 & 7,28 & 7,19 \\
\hline & Média & 8,08 & 3,19 & 25 & 10 & 7,19 & 7,15 \\
\hline
\end{tabular}

\begin{tabular}{|c|c|c|c|c|c|c|c|}
\hline \multicolumn{8}{|c|}{ Configuração A [PAC] 4,8 mg. $\mathrm{L}^{-1}$} \\
\hline \multirow{2}{*}{ Campo } & \multirow{2}{*}{ Triplicata } & \multicolumn{2}{|c|}{ Turbidez (NTU) } & \multicolumn{2}{|c|}{ Cor Aparente (uH) } & \multicolumn{2}{|c|}{ pH } \\
\hline & & Inicial & Final & Inicial & Final & Inicial & Final \\
\hline \multirow{4}{*}{0} & 1 & 6,00 & 1,50 & 25 & 10 & 7,59 & 7,35 \\
\hline & 2 & 9,80 & 1,09 & 25 & 10 & 7,11 & 7,10 \\
\hline & 3 & 9,52 & 1,29 & 40 & 10 & 7,19 & 7,12 \\
\hline & Média & 8,44 & 1,29 & 30 & 10 & 7,30 & 7,19 \\
\hline \multirow{4}{*}{8} & 1 & 6,42 & 1,07 & 25 & 10 & 7,12 & 7,02 \\
\hline & 2 & 7,81 & 1,24 & 25 & 10 & 7,28 & 7,16 \\
\hline & 3 & 7,60 & 1,31 & 35 & 10 & 7,22 & 7,19 \\
\hline & Média & 7,28 & 1,20 & 28 & 10 & 7,21 & 7,12 \\
\hline \multirow{4}{*}{12} & 1 & 5,15 & 1,25 & 35 & 10 & 7,39 & 7,20 \\
\hline & 2 & 9,18 & 1,42 & 35 & 10 & 7,29 & 7,15 \\
\hline & 3 & 8,83 & 1,35 & 35 & 10 & 7,29 & 7,14 \\
\hline & Média & 7,72 & 1,34 & 35 & 10 & 7,32 & 7,16 \\
\hline \multirow{4}{*}{24} & 1 & 5,98 & 1,36 & 25 & 10 & 7,65 & 7,17 \\
\hline & 2 & 7,97 & 1,11 & 25 & 10 & 7,30 & 7,18 \\
\hline & 3 & 6,96 & 1,42 & 30 & 10 & 7,30 & 7,18 \\
\hline & Média & 6,97 & 1,30 & 27 & 10 & 7,42 & 7,18 \\
\hline
\end{tabular}




\begin{tabular}{|c|c|c|c|c|c|c|c|}
\hline \multicolumn{8}{|c|}{ Configuração A [PAC] $9,6 \mathrm{mg} \cdot \mathrm{L}^{-1}$} \\
\hline \multirow{2}{*}{ Campo } & \multirow{2}{*}{ Triplicata } & \multicolumn{2}{|c|}{ Turbidez (NTU) } & \multicolumn{2}{|c|}{ Cor Aparente (uH) } & \multicolumn{2}{|c|}{$\mathrm{pH}$} \\
\hline & & Inicial & Final & Inicial & Final & Inicial & Final \\
\hline \multirow{4}{*}{0} & 1 & 6,02 & 0,74 & 30 & 10 & 7,17 & 6,99 \\
\hline & 2 & 6,18 & 0,72 & 25 & 10 & 7,35 & 7,20 \\
\hline & 3 & 7,01 & 0,75 & 25 & 10 & 7,35 & 7,20 \\
\hline & Média & 6,40 & 0,73 & 27 & 10 & 7,29 & 7,13 \\
\hline \multirow{4}{*}{8} & 1 & 6,79 & 0,67 & 30 & 5 & 7,17 & 6,95 \\
\hline & 2 & 6,76 & 0,69 & 30 & 10 & 7,17 & 7,11 \\
\hline & 3 & 7,22 & 0,50 & 25 & 10 & 7,09 & 7,06 \\
\hline & Média & 6,92 & 0,62 & 28 & 8 & 7,14 & 7,04 \\
\hline \multirow{4}{*}{12} & 1 & 6,35 & 0,79 & 30 & 5 & 7,29 & 7,06 \\
\hline & 2 & 5,65 & 0,47 & 30 & 5 & 7,16 & 7,05 \\
\hline & 3 & 7,15 & 0,51 & 30 & 5 & 7,09 & 7,05 \\
\hline & Média & 6,38 & 0,59 & 30 & 5 & 7,18 & 7,05 \\
\hline \multirow{4}{*}{24} & 1 & 6,34 & 0,74 & 30 & 5 & 7,29 & 7,09 \\
\hline & 2 & 6,03 & 0,48 & 25 & 5 & 7,25 & 6,88 \\
\hline & 3 & 9,65 & 0,48 & 25 & 5 & 6,97 & 6,88 \\
\hline & Média & 7,34 & 0,56 & 27 & 5 & 7,17 & 6,95 \\
\hline
\end{tabular}

\begin{tabular}{|c|c|c|c|c|c|c|c|}
\hline \multicolumn{8}{|c|}{ Configuração A [PAC] 14,4 mg. $\mathrm{L}^{-1}$} \\
\hline \multirow{2}{*}{ Campo } & \multirow{2}{*}{ Triplicata } & \multicolumn{2}{|c|}{ Turbidez (NTU) } & \multicolumn{2}{|c|}{ Cor Aparente (uH) } & \multicolumn{2}{|c|}{$\mathrm{pH}$} \\
\hline & & Inicial & Final & Inicial & Final & Inicial & Final \\
\hline \multirow{4}{*}{0} & 1 & 5,57 & 0,44 & 25 & 5 & 7,26 & 7,13 \\
\hline & 2 & 6,99 & 0,45 & 20 & 5 & 7,28 & 7,04 \\
\hline & 3 & 7,39 & 0,50 & 20 & 5 & 7,16 & 7,03 \\
\hline & Média & 6,65 & 0,46 & 22 & 5 & 7,23 & 7,07 \\
\hline \multirow{4}{*}{8} & 1 & 6,92 & 0,36 & 20 & 5 & 7,27 & 7,12 \\
\hline & 2 & 6,28 & 0,15 & 25 & 5 & 7,22 & 6,97 \\
\hline & 3 & 8,52 & 0,45 & 20 & 5 & 7,16 & 6,99 \\
\hline & Média & 7,24 & 0,32 & 22 & 5 & 7,22 & 7,03 \\
\hline \multirow{4}{*}{12} & 1 & 7,14 & 0,39 & 20 & 5 & 7,25 & 7,15 \\
\hline & 2 & 7,35 & 0,39 & 30 & 5 & 7,05 & 7,01 \\
\hline & 3 & 5,79 & 0,38 & 30 & 5 & 7,15 & 6,99 \\
\hline & Média & 6,76 & 0,39 & 27 & 5 & 7,15 & 7,05 \\
\hline \multirow{4}{*}{24} & 1 & 7,54 & 0,45 & 20 & 5 & 7,20 & 6,90 \\
\hline & 2 & 6,57 & 0,33 & 30 & 5 & 7,05 & 6,89 \\
\hline & 3 & 6,96 & 0,37 & 30 & 5 & 7,10 & 6,91 \\
\hline & Média & 7,02 & 0,38 & 27 & 5 & 7,12 & 6,90 \\
\hline
\end{tabular}

\begin{tabular}{|c|c|c|c|c|c|c|c|}
\hline \multicolumn{8}{|c|}{ Configuração A [PAC] 19,2 mg. L-1 } \\
\hline \multirow{2}{*}{ Campo } & \multirow{2}{*}{ Triplicata } & \multicolumn{2}{|c|}{ Turbidez (NTU) } & \multicolumn{2}{|c|}{ Cor Aparente (uH) } & \multicolumn{2}{|c|}{$\mathrm{pH}$} \\
\hline & & Inicial & Final & Inicial & Final & Inicial & Final \\
\hline \multirow{4}{*}{0} & 1 & 6,51 & 0,34 & 30 & 5 & 7,16 & 6,98 \\
\hline & 2 & 5,58 & 0,37 & 25 & 5 & 7,25 & 7,05 \\
\hline & 3 & 8,73 & 0,34 & 30 & 10 & 7,00 & 6,68 \\
\hline & Média & 6,94 & 0,35 & 28 & 7 & 7,14 & 6,90 \\
\hline \multirow{4}{*}{8} & 1 & 5,73 & 0,27 & 25 & 5 & 7,14 & 6,99 \\
\hline & 2 & 5,86 & 0,27 & 25 & 5 & 7,25 & 7,02 \\
\hline & 3 & 6,12 & 0,30 & 35 & 10 & 7,12 & 6,62 \\
\hline & Média & 5,90 & 0,28 & 28 & 7 & 7,17 & 6,88 \\
\hline \multirow{4}{*}{12} & 1 & 7,00 & 0,33 & 30 & 5 & 7,16 & 6,95 \\
\hline & 2 & 6,90 & 0,26 & 30 & 5 & 7,12 & 6,71 \\
\hline & 3 & 6,51 & 0,30 & 30 & 5 & 7,12 & 6,88 \\
\hline & Média & 6,80 & 0,30 & 30 & 5 & 7,13 & 6,85 \\
\hline \multirow{4}{*}{24} & 1 & 5,49 & 0,34 & 25 & 5 & 7,22 & 6,80 \\
\hline & 2 & 8,43 & 0,35 & 30 & 5 & 7,28 & 6,81 \\
\hline & 3 & 7,25 & 0,32 & 35 & 5 & 7,35 & 6,80 \\
\hline & Média & 7,06 & 0,34 & 30 & 5 & 7,28 & 6,80 \\
\hline
\end{tabular}




\begin{tabular}{|c|c|c|c|c|c|c|c|}
\hline \multicolumn{8}{|c|}{ Configuração A [PAC] 24,0 mg. $\mathrm{L}^{-1}$} \\
\hline \multirow{2}{*}{ Campo } & \multirow{2}{*}{ Triplicata } & \multicolumn{2}{|c|}{ Turbidez (NTU) } & \multicolumn{2}{|c|}{ Cor Aparente (uH) } & \multicolumn{2}{|c|}{ pH } \\
\hline & & Inicial & Final & Inicial & Final & Inicial & Final \\
\hline \multirow{4}{*}{0} & 1 & 5,86 & 0,34 & 30 & 5 & 7,31 & 7,04 \\
\hline & 2 & 6,90 & 0,25 & 20 & 5 & 7,11 & 6,92 \\
\hline & 3 & 8,39 & 0,31 & 35 & 10 & 7,65 & 7,04 \\
\hline & Média & 7,05 & 0,30 & 28 & 7 & 7,36 & 7,00 \\
\hline \multirow{4}{*}{8} & 1 & 5,30 & 0,08 & 20 & 5 & 7,22 & 7,06 \\
\hline & 2 & 6,02 & 0,22 & 30 & 5 & 7,29 & 7,14 \\
\hline & 3 & 7,14 & 0,31 & 25 & 10 & 7,57 & 6,82 \\
\hline & Média & 6,15 & 0,20 & 25 & 7 & 7,36 & 7,01 \\
\hline \multirow{4}{*}{12} & 1 & 5,75 & 0,26 & 20 & 5 & 7,30 & 7,05 \\
\hline & 2 & 7,67 & 0,28 & 30 & 5 & 7,18 & 6,79 \\
\hline & 3 & 7,32 & 0,34 & 25 & 10 & 7,68 & 6,96 \\
\hline & Média & 6,91 & 0,29 & 25 & 7 & 7,39 & 6,93 \\
\hline \multirow{4}{*}{24} & 1 & 6,62 & 0,30 & 20 & 5 & 7,30 & 6,88 \\
\hline & 2 & 7,34 & 0,27 & 30 & 5 & 7,58 & 6,94 \\
\hline & 3 & 7,87 & 0,30 & 30 & 10 & 7,69 & 6,98 \\
\hline & Média & 7,27 & 0,29 & 27 & 7 & 7,52 & 6,93 \\
\hline
\end{tabular}

\begin{tabular}{|c|c|c|c|c|c|c|c|}
\hline \multicolumn{8}{|c|}{ Configuração B [PAC] 4,8 mg. $\mathrm{L}^{-1}$} \\
\hline \multirow{2}{*}{ Campo } & \multirow{2}{*}{ Triplicata } & \multicolumn{2}{|c|}{ Turbidez (NTU) } & \multicolumn{2}{|c|}{ Cor Aparente (uH) } & \multicolumn{2}{|c|}{ pH } \\
\hline & & Inicial & Final & Inicial & Final & Inicial & Final \\
\hline \multirow{4}{*}{0} & 1 & 6,82 & 2,06 & 30 & 10 & 7,39 & 7,30 \\
\hline & 2 & 12,85 & 1,94 & 30 & 10 & 7,75 & 7,19 \\
\hline & 3 & 9,26 & 1,90 & 30 & 10 & 7,29 & 7,30 \\
\hline & Média & 9,64 & 1,96 & 30 & 10 & 7,48 & 7,26 \\
\hline \multirow{4}{*}{8} & 1 & 8,71 & 2,00 & 30 & 10 & 7,66 & 7,61 \\
\hline & 2 & 7,96 & 1,68 & 30 & 10 & 7,82 & 7,21 \\
\hline & 3 & 8,85 & 1,76 & 25 & 10 & 7,29 & 7,20 \\
\hline & Média & 8,51 & 1,81 & 28 & 10 & 7,59 & 7,34 \\
\hline \multirow{4}{*}{12} & 1 & 12,90 & 2,22 & 30 & 10 & 7,46 & 7,09 \\
\hline & 2 & 10,90 & 1,96 & 30 & 10 & 7,46 & 7,15 \\
\hline & 3 & 8,34 & 1,72 & 25 & 10 & 7,29 & 7,18 \\
\hline & Média & 10,71 & 1,97 & 28 & 10 & 7,40 & 7,14 \\
\hline \multirow{4}{*}{24} & 1 & 9,26 & 1,81 & 30 & 10 & 7,46 & 7,21 \\
\hline & 2 & 10,78 & 1,90 & 30 & 10 & 7,46 & 7,03 \\
\hline & 3 & 8,73 & 1,59 & 30 & 10 & 7,54 & 7,21 \\
\hline & Média & 9,59 & 1,77 & 30 & 10 & 7,49 & 7,15 \\
\hline
\end{tabular}

\begin{tabular}{|c|c|c|c|c|c|c|c|}
\hline \multicolumn{8}{|c|}{ Configuração B [PAC] 9,6 mg.L-1 } \\
\hline \multirow{2}{*}{ Campo } & \multirow{2}{*}{ Triplicata } & \multicolumn{2}{|c|}{ Turbidez (NTU) } & \multicolumn{2}{|c|}{ Cor Aparente (uH) } & \multicolumn{2}{|c|}{ pH } \\
\hline & & Inicial & Final & Inicial & Final & Inicial & Final \\
\hline \multirow{4}{*}{0} & 1 & 7,28 & 0,65 & 30 & 10 & 7,17 & 6,99 \\
\hline & 2 & 7,28 & 0,72 & 25 & 10 & 7,35 & 7,20 \\
\hline & 3 & 6,47 & 0,71 & 25 & 10 & 7,35 & 7,20 \\
\hline & Média & 7,01 & 0,69 & 27 & 10 & 7,29 & 7,13 \\
\hline \multirow{4}{*}{8} & 1 & 7,28 & 0,74 & 30 & 5 & 7,17 & 6,95 \\
\hline & 2 & 8,10 & 0,57 & 30 & 10 & 7,17 & 7,11 \\
\hline & 3 & 7,62 & 0,64 & 25 & 10 & 7,09 & 7,06 \\
\hline & Média & 7,66 & 0,65 & 28 & 8 & 7,14 & 7,04 \\
\hline \multirow{4}{*}{12} & 1 & 7,78 & 0,27 & 30 & 5 & 7,29 & 7,06 \\
\hline & 2 & 8,67 & 0,43 & 30 & 5 & 7,16 & 7,05 \\
\hline & 3 & 7,62 & 0,60 & 30 & 5 & 7,09 & 7,05 \\
\hline & Média & 8,02 & 0,43 & 30 & 5 & 7,18 & 7,05 \\
\hline \multirow{4}{*}{24} & 1 & 8,48 & 0,72 & 30 & 5 & 7,29 & 7,09 \\
\hline & 2 & 7,48 & 0,75 & 25 & 5 & 7,25 & 6,88 \\
\hline & 3 & 7,62 & 0,64 & 25 & 5 & 6,97 & 6,88 \\
\hline & Média & 7,86 & 0,70 & 27 & 5 & 7,17 & 6,95 \\
\hline
\end{tabular}




\begin{tabular}{|c|c|c|c|c|c|c|c|}
\hline \multicolumn{8}{|c|}{ Configuração B [PAC] 14,4 mg. L-1 } \\
\hline \multirow{2}{*}{ Campo } & \multirow{2}{*}{ Triplicata } & \multicolumn{2}{|c|}{ Turbidez (NTU) } & \multicolumn{2}{|c|}{ Cor Aparente (uH) } & \multicolumn{2}{|c|}{ pH } \\
\hline & & Inicial & Final & Inicial & Final & Inicial & Final \\
\hline \multirow{4}{*}{0} & 1 & 5,57 & 0,44 & 30 & 10 & 7,12 & 6,98 \\
\hline & 2 & 6,99 & 0,45 & 30 & 10 & 7,12 & 6,98 \\
\hline & 3 & 7,39 & 0,50 & 40 & 10 & 7,28 & 7,00 \\
\hline & Média & 6,65 & 0,46 & 33 & 10 & 7,17 & 6,99 \\
\hline \multirow{4}{*}{8} & 1 & 6,92 & 0,36 & 25 & 10 & 7,31 & 7,04 \\
\hline & 2 & 6,28 & 0,15 & 25 & 10 & 7,31 & 7,03 \\
\hline & 3 & 8,52 & 0,45 & 40 & 10 & 7,28 & 7,01 \\
\hline & Média & 7,24 & 0,32 & 30 & 10 & 7,30 & 7,03 \\
\hline \multirow{4}{*}{12} & 1 & 7,14 & 0,39 & 25 & 10 & 7,31 & 6,42 \\
\hline & 2 & 7,35 & 0,39 & 25 & 10 & 7,31 & 6,80 \\
\hline & 3 & 5,79 & 0,38 & 25 & 10 & 7,29 & 6,64 \\
\hline & Média & 6,76 & 0,39 & 25 & 10 & 7,30 & 6,62 \\
\hline \multirow{4}{*}{24} & 1 & 7,54 & 0,45 & 25 & 10 & 7,31 & 6,43 \\
\hline & 2 & 6,57 & 0,33 & 25 & 10 & 7,52 & 6,50 \\
\hline & 3 & 6,96 & 0,37 & 25 & 10 & 7,29 & 6,71 \\
\hline & Média & 7,02 & 0,38 & 25 & 10 & 7,37 & 6,55 \\
\hline
\end{tabular}

\begin{tabular}{|c|c|c|c|c|c|c|c|}
\hline \multicolumn{8}{|c|}{ Configuração B [PAC] 19,2 mg. L-1 } \\
\hline \multirow{2}{*}{ Campo } & \multirow{2}{*}{ Triplicata } & \multicolumn{2}{|c|}{ Turbidez (NTU) } & \multicolumn{2}{|c|}{ Cor Aparente (uH) } & \multicolumn{2}{|c|}{$\mathrm{pH}$} \\
\hline & & Inicial & Final & Inicial & Final & Inicial & Final \\
\hline \multirow{4}{*}{0} & 1 & 7,21 & 0,29 & 25 & 5 & 7,52 & 7,02 \\
\hline & 2 & 7,21 & 0,30 & 25 & 10 & 7,52 & 7,08 \\
\hline & 3 & 8,39 & 0,40 & 25 & 10 & 7,29 & 6,93 \\
\hline & Média & 7,60 & 0,33 & 25 & 8 & 7,44 & 7,01 \\
\hline \multirow{4}{*}{8} & 1 & 7,21 & 0,32 & 25 & 5 & 7,52 & 7,15 \\
\hline & 2 & 7,21 & 0,37 & 25 & 5 & 7,52 & 7,11 \\
\hline & 3 & 8,39 & 0,45 & 25 & 5 & 7,29 & 6,94 \\
\hline & Média & 7,60 & 0,38 & 25 & 5 & 7,44 & 7,07 \\
\hline \multirow{4}{*}{12} & 1 & 8,41 & 0,33 & 30 & 5 & 7,31 & 6,96 \\
\hline & 2 & 8,94 & 0,34 & 25 & 5 & 7,31 & 6,95 \\
\hline & 3 & 8,39 & 0,42 & 25 & 5 & 7,29 & 6,88 \\
\hline & Média & 8,58 & 0,36 & 27 & 5 & 7,30 & 6,93 \\
\hline \multirow{4}{*}{24} & 1 & 11,16 & 0,31 & 25 & 5 & 7,31 & 6,96 \\
\hline & 2 & 10,02 & 0,34 & 25 & 5 & 7,31 & 6,94 \\
\hline & 3 & 9,84 & 0,29 & 30 & 5 & 6,95 & 6,77 \\
\hline & Média & 10,34 & 0,31 & 27 & 5 & 7,19 & 6,89 \\
\hline
\end{tabular}

\begin{tabular}{|c|c|c|c|c|c|c|c|}
\hline \multicolumn{8}{|c|}{ Configuração B [PAC] $24,0 \mathrm{mg} \cdot \mathrm{L}^{-1}$} \\
\hline \multirow{2}{*}{ Campo } & \multirow{2}{*}{ Triplicata } & \multicolumn{2}{|c|}{ Turbidez (NTU) } & \multicolumn{2}{|c|}{ Cor Aparente (uH) } & \multicolumn{2}{|c|}{ pH } \\
\hline & & Inicial & Final & Inicial & Final & Inicial & Final \\
\hline \multirow{4}{*}{0} & 1 & 6,34 & 0,30 & 30 & 5 & 7,49 & 6,43 \\
\hline & 2 & 6,34 & 0,28 & 30 & 10 & 7,49 & 6,64 \\
\hline & 3 & 9,84 & 0,31 & 30 & 5 & 6,95 & 5,62 \\
\hline & Média & 7,51 & 0,30 & 30 & 7 & 7,31 & 6,23 \\
\hline \multirow{4}{*}{8} & 1 & 6,34 & 0,31 & 30 & 5 & 7,49 & 7,25 \\
\hline & 2 & 6,34 & 0,32 & 30 & 5 & 7,49 & 6,43 \\
\hline & 3 & 9,84 & 0,28 & 30 & 5 & 6,95 & 6,74 \\
\hline & Média & 7,51 & 0,30 & 30 & 5 & 7,31 & 6,81 \\
\hline \multirow{4}{*}{12} & 1 & 6,34 & 0,29 & 30 & 5 & 7,49 & 6,71 \\
\hline & 2 & 8,96 & 0,32 & 30 & 5 & 7,12 & 6,69 \\
\hline & 3 & 9,77 & 0,26 & 30 & 5 & 7,12 & 6,19 \\
\hline & Média & 8,36 & 0,29 & 30 & 5 & 7,24 & 6,53 \\
\hline \multirow{4}{*}{24} & 1 & 8,96 & 0,25 & 30 & 5 & 7,12 & 5,99 \\
\hline & 2 & 8,96 & 0,25 & 30 & 5 & 7,12 & 5,95 \\
\hline & 3 & 8,96 & 0,18 & 30 & 5 & 7,12 & 6,12 \\
\hline & Média & 8,96 & 0,22 & 30 & 5 & 7,12 & 6,02 \\
\hline
\end{tabular}

